\title{
Free Software Package SIGMA_FW for Numerical Simulation of Hydrodynamics and Heat Transfer
}

\author{
Alexander A. Dekterev $v^{\mathrm{a}, \mathrm{b}}$, \\ Kirill Yu. Litvintsev ${ }^{a}$, Andrey A. Gavrilova, \\ Egor B. Kharlamov ${ }^{a}$ and Sergey A. Filimonov ${ }^{b}$ \\ ${ }^{a}$ S.S. Kutateladze Institute of Thermophysics $S B R A S$ \\ 1 Akademika Lavrentieva, Novosibirsk, 630090, Russia, \\ ${ }^{b}$ Siberian Federal University \\ 79 Svobodny, Krasnoyarsk, 660041, Russia
}

The Department of Thermophysics of the Siberian Federal University and Krasnoyarsk Subsidiary Institute of Thermophysics SB RAS are developing a freely distributable "SigmaFW" software package for numerical simulation of the hydrodynamics and heat and mass transfer problems. It is assumed that the software package will be used in scientific and educational institutions as well as industrial enterprises in Russian Federation. The "SigmaFW" software package contains the necessary tools for building grids, carrying out multi-threaded calculation, and visual analysis of the results.

Keywords: software package, mathematical modeling, computational fluid dynamics, heat and mass transfer.

Citation: Dekterev A.A., Litvintsev K.Yu., Gavrilov A.A., Kharlamov E.B., Filimonov S.A. Free software package SIGMA_FW for numerical simulation of hydrodynamics and heat transfer, J. Sib. Fed. Univ. Eng. technol., 2017, 10(4), 534542. DOI: 10.17516/1999-494X-2017-10-4-534-542.

(C) Siberian Federal University. All rights reserved

* Corresponding author E-mail address: dekterev@mail.ru 


\title{
Свободно распространяемый
}

\section{программный комплекс SIGMA_FW}

\section{для моделирования гидродинамики и теплообмена}

\author{
А.А. Дектерев ${ }^{\mathrm{a}, \tilde{\sigma}}$, К.Ю. Литвинцев ${ }^{\mathrm{a}}$, \\ А.А. Гаврилов ${ }^{\text {a, }}$, Е.Б. Харламов ${ }^{\text {a }}$, С.А. Филимонов ${ }^{\sigma}$ \\ ${ }^{a}$ Институт теплофизики им. С.С. Кутателадзе СО РАН \\ Россия, 630090, Новосибирск, пр. Академика Лаврентьева, 1 \\ ${ }^{6}$ Сибирский федеральный университет \\ Россия, 660041, Красноярск, пр. Свободный,79
}

Накафедретеплофизики СФУи К Касноярском филиале ИТСОРАНразрабатывается свободно распространяемый программный комплекс «SigmaFW» для численного моделирования задач гидрогазодинамики и тепломассообмена, который предполагается использовать в научных и образовательных организациях и промышленных предприятиях России. Программный комплекс «SigmaFW» содержит необходимые инструменты для построения сеток, проведения многопоточных (параллельных) вычислений и визуального анализа результатов расчета.

Ключевые слова: программный комплекс, математическое моделирование, вычислительная гидродинамика, тепломассообмен.

\section{Вступление}

Математическое моделирование процессов гидродинамики и тепломассопереноса как один из важных инструментов для проведения научных фундаментальных и прикладных исследований, анализа технологических процессов, оптимизации и модернизации технологических комплексов и установок успешно применяется во всем мире. При этом в российских вузах, проектных организациях и НИИ уровень использования данного инструмента все еще достаточно низкий. Это обусловлено несколькими причинами, наиболее значимыми из которых являются финансовые затраты, связанные с покупкой и поддержкой соответствующего программного обеспечения, и отсутствие достаточного количества квалифицированных специалистов по численному моделированию процессов гидродинамики и тепломассопереноса. Использование наиболее развитых зарубежных программных комплексов ANSYS CFD или STAR-CCM+ требует вложений десятки - сотни тысяч долларов. Единственный российский коммерческий программный CFD-комплекс «FlowVision» обладает меньшими функциональными возможностями. Как и во всем мире в РФ развиваются отраслевые и университетские программы. Наиболее известный из отраслевых программных комплексов - это «LOGOS», ориентированный на задачи военно-промышленного комплекса и задачи атомной энергетики. На данный момент «LOGOS» не доступен для общего использования. В качестве примера университетских кодов можно привести разработанные в Санкт-Петербурге программы «VP2/3» и «SINF». Особенность университетских кодов в том, что они предназначены в основном для внутреннего использования. За рубежом существует еще ряд открытых кодов (например, «OpenFoAM»), которые также используются российскими исследователями, но их освоение требует значительно большего времени и более высокой квалификации пользователя. 
Таким образом, актуален вопрос создания для научных и образовательных организаций и промышленных предприятий в РФ доступного (свободно распространяемого) полноценного программного комплекса для моделирования задач гидрогазодинамики и тепломассообмена.

Авторами публикации длительное время развивается университетский код «SigmaFlow» [1], ядро которого используется для создания свободно распространяемого программного комплекса «SigmaFW».

\section{Краткое описание программного комплекса}

Программный комплекс «SigmaFW» состоит из трех основных модулей: модуль подготовки расчета, расчетный модуль, модуль анализа результатов. Модуль подготовки расчета включает в себя генератор сетки и модуль задания граничных и начальных условий, теплофизических параметров и параметров математической модели. В генераторе сетки дискретизация расчетной области проводится на основе сторонней (импортируемой) геометрии, предварительно подготовленной во внешней CAD-системе. Процесс генерации сетки основан на восьмеричном разбиении пространства геометрии. Сначала на основе размеров геометрического объекта строится один пространственный элемент (куб), в которой заключен объект (рис. 1). Этот элемент, в свою очередь, разбивается на восемь элементов (кубов), и далее процесс повторяется для каждого элемента. Момент, на котором получен первый элемент, будет соответствовать первому уровню разбиения. Выбирая части для последующего разбиения и момент прекращения разбиения для элемента, добиваются нужной детализации будущей сетки в необходимых областях геометрического объекта. Таким образом, для расчетной области строится октодерево с необходимой глубиной разбиения внутри и на границах геометрии. Полученные листовые элементы октодерева определяют узлы будущей сетки. В процессе разбиения элементы делятся на три группы: элементы, выходящие за границу объекта; элементы внутри объекта; элементы, пересекаемые границей объекта. Интерфейс пользователя позволяет задавать нужный уровень разбиения внутри объекта, на его границах, на расстоянии от выбранной границы или в замкнутой прямоугольной области, заданной пользователем. На основе получившегося пространственного распределения элементов далее строится конечная сетка. В результате формируется неструктурированная гибридная сетка (рис. 1), включающая в себе шестигранные элементы (кубы), расположенные в основном внутри области объекта, и многогранные элементы (пирамиды, призмы, тетраэдры), находящиеся в переходных областях и на границах объекта.

В расчетном модуле реализованы математические модели, описывающие стационарные и нестационарные ламинарные и турбулентные одно- и многокомпонентные течения сопряжённого и лучистого теплообмена. Дискретизация уравнений гидродинамики осуществляется методом контрольного объема. Для моделирования турбулентности газовой фазы стационарных и нестационарных течений используется осреднение по Рейнольдсу уравнений Навье-Стокса (RANS). Для замыкания уравнений Рейнольдса применяются модели как с использованием гипотезы вихревой вязкости, так и на основе уравнений переноса для рейнольдсовых напряжений. В программе реализованы две модели вихревой вязкости, позволяющие разрешать пограничные турбулентные слои, двухпараметрическая k- $\omega$ SST-модель [2] и четырёх параметрическая модель k- $\varepsilon-\mathrm{f}-\zeta$ [3]. Математическая модель переноса излучения основана на методе конечных объемов (МКО) [4]. Оптические свойства среды описываются WSGG (Weighted Sum 

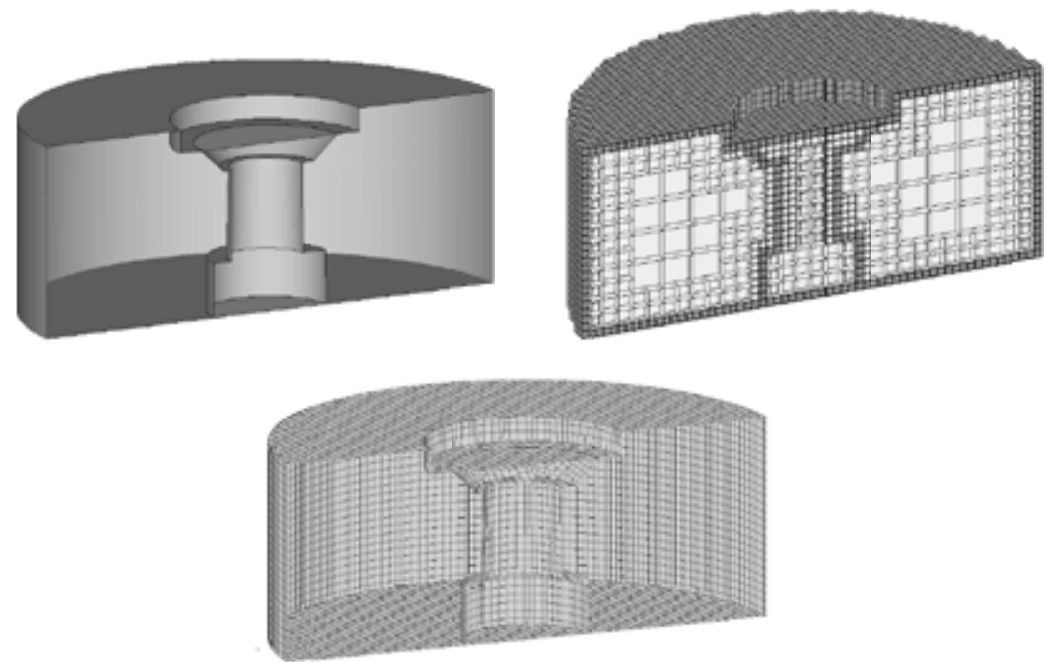

Рис. 1. Создание из CAD геометрии предварительного разбиения расчетной области и переход к построению итоговой сетки

of Gray Gas) моделями, обеспечивающими приемлемую точность для большого класса прикладных задач, связанных со сжиганием углеродного топлива. Кроме этого, расчетный модуль поддерживает многопоточные вычисления. Распараллеливание алгоритмов вычислительной гидродинамики основано на декомпозиции расчетной области и использовании стандарта MPI. SigmaFW позволяет выполнять параллельные расчеты как на персональных ЭВМ, так и на современных кластерных системах с операционными системами Windows и Linux.

Для проведения анализа результатов расчета программный комплекс включает в себя модуль 3D-визуализации, позволяющий отображать скалярные значения физических величин в произвольных сечениях в виде изоповерхностей и графиков вдоль выбранных отрезков, значения векторных физических величин в произвольных сечениях - в виде поля векторов, геометрию расчетной области и ее сеточную дискретизацию.

Документация на программу содержит обучающие примеры ряда задач течения и теплообмена.

\section{Примеры решения тестовых и прикладных задач}

В качестве одного из примеров рассмотрено обтекание профиля гидрокрыла с углом атаки $3^{\circ}$ в ограниченном канале [5]. При численном моделировании обтекания гидрокрыла

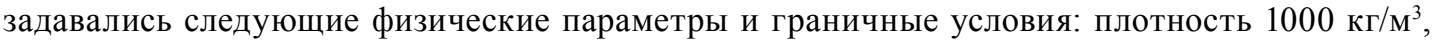
динамическая вязкость $10^{-6}$ Па·с, на входе массовый расход 0.025 кг/с с ударным профилем (среднерасходная скорость набегающего 1 м/с), на выходе свободный массовый расход. Турбулентность описывается моделью переноса рейнольдсовых напряжений. На рис. 2 представлена расчетная сетка вблизи крыла. Проверка результатов моделирования осуществлялась путем сравнения с экспериментом [5]. Для сравнения используются распределения скорости вдоль вертикальных линий (OY), заданных положением вдоль продольной оси (ОХ) от передней кромки крыла (при нулевом угле атаки): $\mathrm{x} / \mathrm{C}=0.25, \mathrm{x} / \mathrm{C}=0.75(\mathrm{C}=0,1 \mathrm{м}-$ длина хорды 
крыла). Продольная компонента скорости сравнивается в безразмерном виде, отнесена к характерной скорости U0, за которую принималась средняя скорость через проходное сечение канала. На рис. 3 показаны графики сравнения расчетной и экспериментальной продольной компоненты скорости.

Во втором примере приводится сравнение результатов расчета поля излучения, полученного с использованием кода «SigmaFW», с данными из работы Yu M.J. [6], в которой использовался метод дискретных ординат. Задача представляет собой закрытый цилиндр, заполненный газовой смесью $\left(\mathrm{H}_{2} \mathrm{O}-20 \%, \mathrm{CO}_{2}-10 \%, \mathrm{~N}_{2}-70 \%\right)$ с однородной температурой. Расчет переноса излучения проводился МКО с 96 дискретными направлениями с использованием WSGG модели расчета коэффициента поглощения [7] (как в работе [6]) и построенной на ее основе модели «серого газа». Сравнение результатов осуществляли по потоку излучения на боковую стенку цилиндра. При применении WSGG модели распределение потока излучения на стенку практически совпадают, а использование приближения «серого газа» очень завышает поток излучения (рис. 4б).

На примере моделирования рабочего процесса внутри вакуумной графитовой электропечи проводился сравнительный расчет теплообмена на основе авторского программного комплекса «SigmaFW» и коммерческого программного комплекса «ANSYS Fluent». Основной технологи-

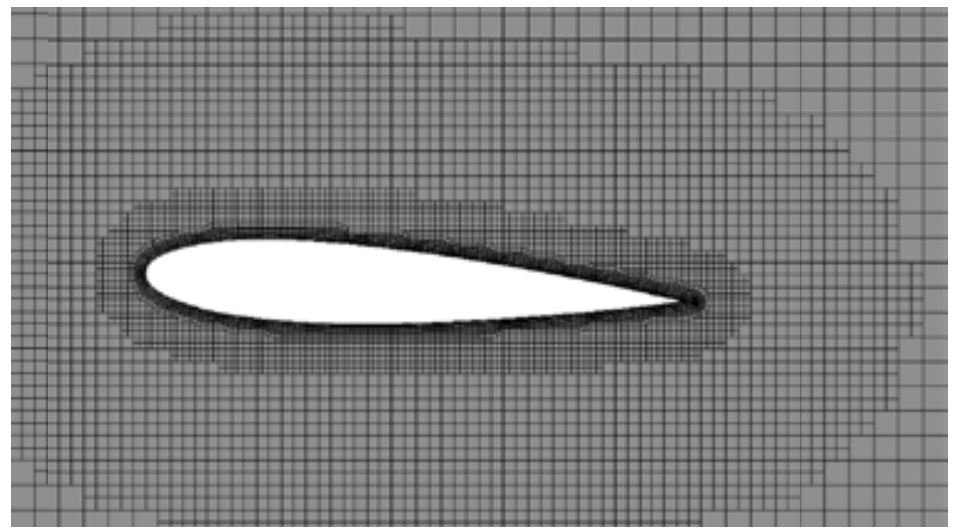

Рис. 2. Расчётная сетка в области гидрокрыла

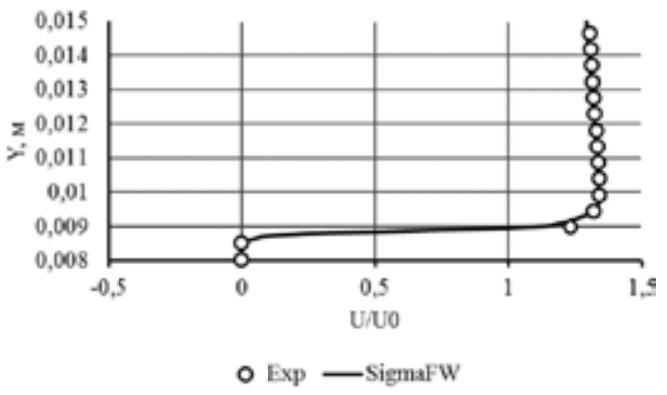

a)

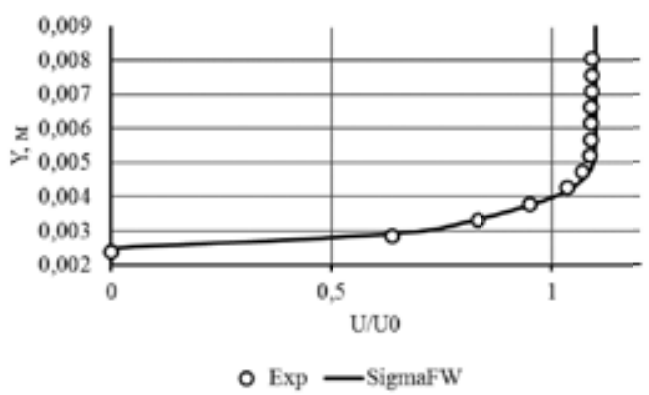

б)

Рис. 3. Сравнение относительной продольной скорости в разных сечениях: $\mathrm{a}-\mathrm{x} / \mathrm{C}=0.25 ; \sigma-\mathrm{x} / \mathrm{C}=0.75$ 
ческий процесс происходит внутри реторты, которая одновременно является и нагревательным элементом печи. Характерное время цикла работы печи составляет один-два дня. Доминирующий механизм теплопереноса в печи - излучение. Течение газов внутри печи ламинарное вследствие низкой плотности газа и малых скоростей $(\mathrm{Re} \sim 10)$. Сетки для «SigmaFW» (630 000 ячеек) и «ANSYS» (850 000 ячеек) были построены на основе импортированной из CAD системы геометрии (рис. 5). Для численного моделирования переноса излучения в ANSYS исполь-

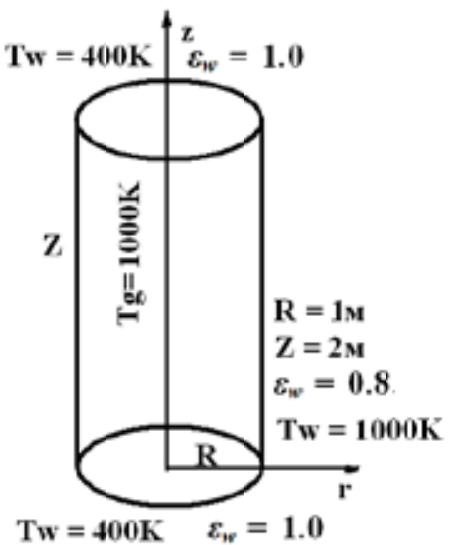

a)

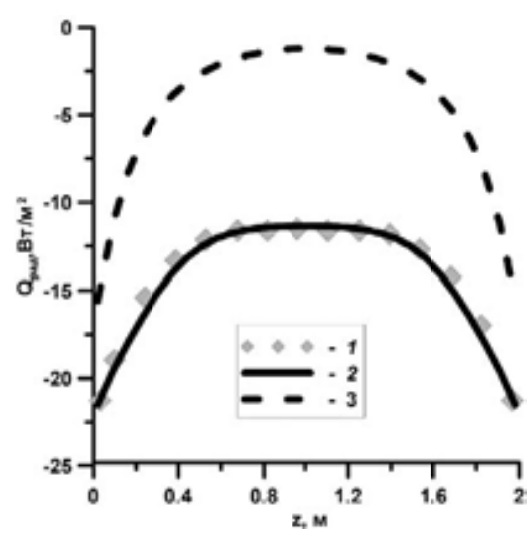

б)

Рис. 4. Замкнутый цилиндр, заполненный смесью излучающих газов: а - описание задачи; б распределение потока излучения на боковую грань цилиндра: 1 - Yu et al, 2000; 2 - SigmaFW (MKO c WSGG); 3 - SigmaFW (MКО с приближением «серого газа»)

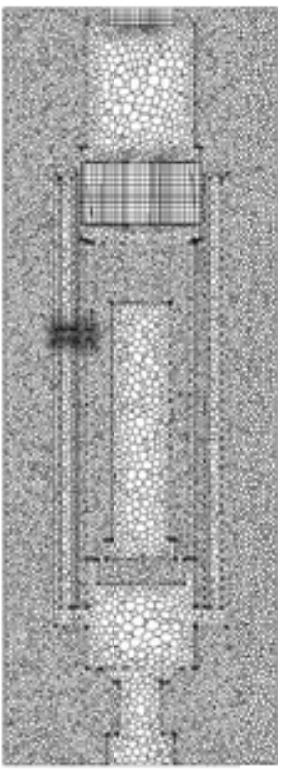

a)

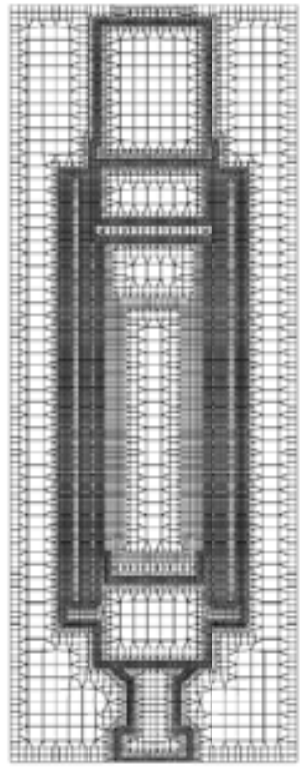

б)

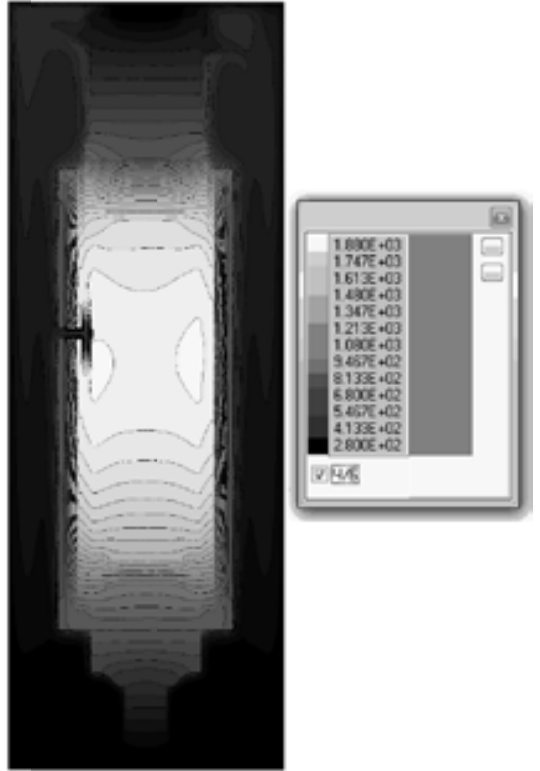

B)

Рис. 5. Расчет электропечи: a - расчетная сетка «ANSYS»; б - расчетная сетка «SigmaFW»; в - поле температур, рассчитанное в «SigmaFW» 
зовался дискретно-ординатный метод, в «SigmaFW»-MKO. Максимальное расхождение по температуре составляет менее 15 К (рис. 6).

Для демонстрации возможностей программы «SigmaFW» моделирования сложных турбулентных течений с теплообменом приведены результаты расчета обтекания компьютерной материнской платы в системном блоке (рис. 7a). Расчётная сетка для этой задачи содержит 1100000 ячеек (рис. 7б). Все компоненты, расположенные на материнской плате: процессор, планки оперативной памяти, конденсаторы, блок питания, - являются источниками тепла. На входе в корпус задается закрученный поток воздуха, создаваемый вентилятором.

Полученное поле температур и траектории движения частиц-маркеров представлены на рис. 8, где видно, что наиболее существенное влияние на структуру потока и процесс теплообмена оказывает радиатор, расположенный напротив вентилятора.

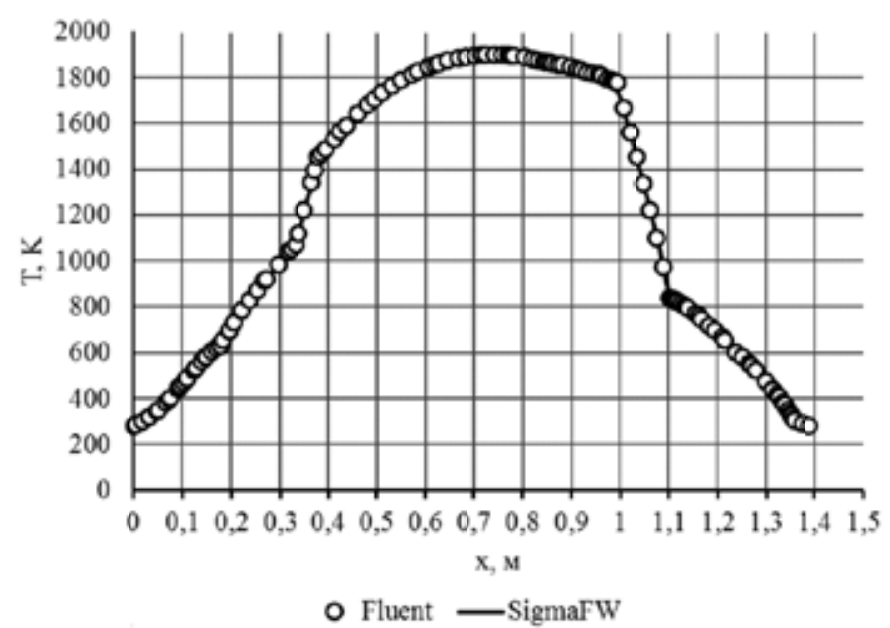

Рис. 6. Сравнение результатов моделирования распределения температуры вдоль диаметра химического реактора на основе программных комплексов «SigmaFW» и «ANSYS»

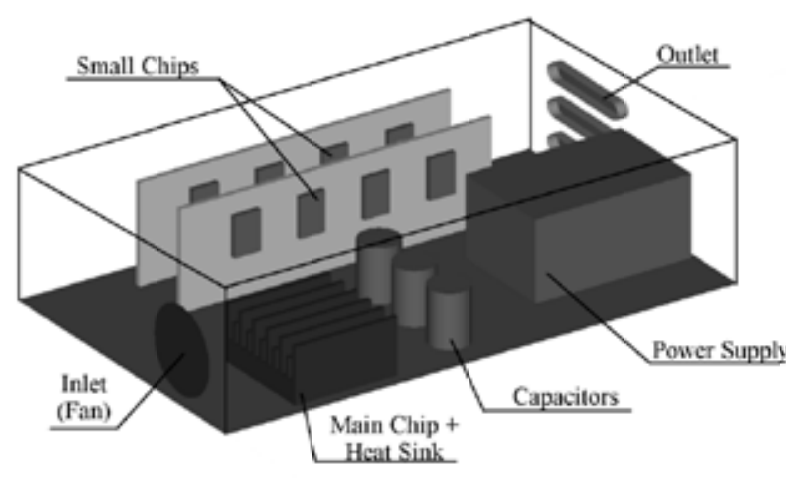

a)

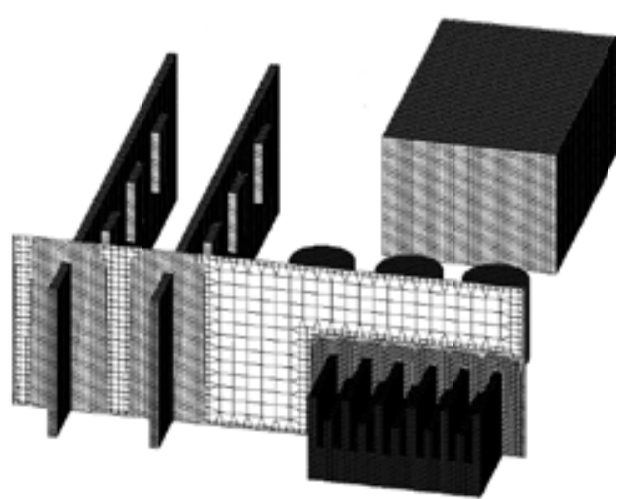

б)

Рис. 7. Геометрия компьютерной материнской платы и расчетная сетка, построенная в программном комплексе «SigmaFW»: a - геометрия; б - сетка 


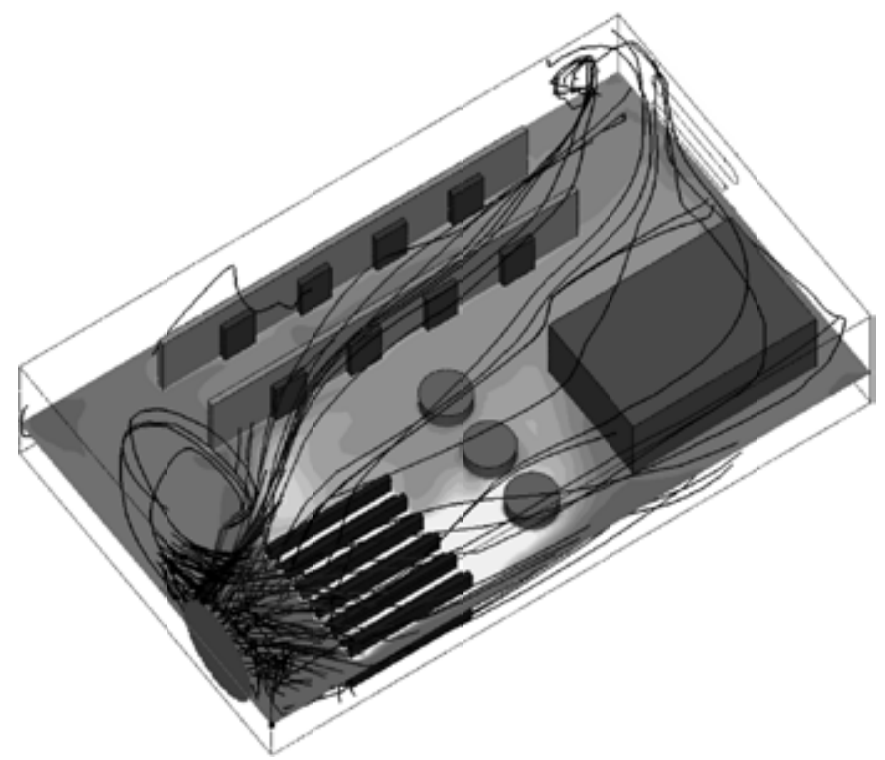

Рис. 8. Результаты расчета аэродинамики и теплообмена в системном блоке. Поле температуры (шкала от 20 до $\left.50{ }^{\circ} \mathrm{C}\right)$ и визуализация потока воздуха

\section{Заключение}

Создан свободно распространяемый программный комплекс «SigmaFW» для решения задач гидродинамики и теплообмена на основе моделирования взаимосвязанных физических процессов, который содержит в себе весь необходимый инструментарий для создания расчетных сеток, проведения многопоточных вычислений и полноценного анализа результатов расчета.

Продемонстрированы возможности «SigmaFW» на примерах решения тестовых и прикладных задач.

Исследование выполнено при финансовой поддержке РФФИ, Правительства Красноярского края, Красноярского краевого фонда поддержки научной и научно-технической деятельности в рамках научных проектов № 16-48-242085 и 16-41-242156 р_офи_м.

\section{Список литературы}

[1] Дектерев А.А., Гаврилов А.А., Минаков А.В. Современные возможности CFD кода SigmaFlow для решения теплофизических задач. Современная наука: исследования, идеи, результаты, технологии, 2010, 2(4), 117-122.

[2] Menter F.R. Zonal two equation $k$ - $\omega$ turbulence models for aerodynamic flows. AIAA Paper. 1993. № 93-2906. 21 p.

[3] Hanjalić K., Popovac M. and Hadžiabdić M. A robust near-wall elliptic relaxation eddy viscosity turbulence model for CFD. Int. J. Heat Fluid Flow, 2004 25(6);1047-1051, .

[4] Chai, J.C., and Patankar, S.V. Finite-Volume Method for Radiation Heat Transfer. Advances in Numerical Heat Transfer. Taylor \& Francis 2000, vol. 2, Chapter 4, 109-138.

$$
-541-
$$


[5] Hanjalic' K. Cavitation on a semicircular leading-edge plate and NACA0015 hydrofoil: visualization and velocity measurement. Thermal Engineering. 2014, Vol. 61, no. 14, 1007-1014.

[6] Smith T.F., Shenand Z.F. and Friedman J.N. Evaluation of Coefficients for the Weighted Sum of Gray Gases Model. Journal of Heat Transfer. 1982, 104, 602-608.

[7] Yu M.J., Baek S.W. and Park J.H. An extension of the weighted sum of fray gases non-gray gas radiation model to a two phase mixture of non-gray gas with particles. Int. J. Heat Mass Transfer. 2000, Vol. 43. 1699-1713. 